\title{
BMJ Open Sustainability drivers and inhibitors for the health system performance improvement projects in selected health facilities in Kenya: a qualitative study
}

\author{
Tecla Chelagat (1) , Gilbert Kokwaro, Joseph Onyango, Jim Rice
}

To cite: Chelagat T,

Kokwaro G, Onyango J, et al.

Sustainability drivers and inhibitors for the health system performance improvement projects in selected health facilities in Kenya: a qualitative study. BMJ Open 2021;11:e035475. doi:10.1136/ bmjopen-2019-035475

- Prepublication history and additional supplemental material for this paper is available online. To view these files, please visit the journal online (http://dx.doi org/10.1136/bmjopen-2019035475).

Received 02 November 2019 Revised 07 July 2020 Accepted 07 August 2020
Check for updates

(c) Author(s) (or their employer(s)) 2021. Re-use permitted under CC BY-NC. No commercial re-use. See rights and permissions. Published by BMJ.

Institute of Healthcare Management, Strathmore University Strathmore Business School, Nairobi, Kenya

Correspondence to

Dr Tecla Chelagat;

tkivuli@strathmore.edu

\section{ABSTRACT}

Objectives To investigate the health managers perceived sustainability status of the health facilities institutional improvement projects and their experiences on factors that facilitated or constrained their maintenance, with intentions of informing relevant strategies or policies in Kenya's health sector.

Methods A qualitative study, nested within a quasiexperimental study. Thirty-three project-teams of health managers were purposively selected and interviewed based on their project implementation success rates post-training. The managers had previously undergone a 9-month leadership training, complimented with facilitybased team coaching around the chosen projects. The training was funded by the US Agency for International Development; however, the implementation of the projects was based on how the participants could innovatively use the existing resource to create a positive change. The projects were housed within 20 public, 9 faith-based and 4 private health facilities in 19 counties in Kenya. The interviews explored the manager's experiences in sustaining the successfully implemented projects within the (24-60 months post-training period). We asked managers to describe factors they perceived enabled or hindered the sustainability of the successfully implemented institutional improvement project. The digitally audio-recorded interviews were transcribed verbatim. Data on barriers and enablers were thematically analysed.

Results Twenty-nine out of the 33 successfully implemented projects reported sustainability within periods ranging from 24 to 60 months post-training. Seven themes related to drivers of sustainability emerged, namely; programme design, stakeholder's buy-in, board members, communication, coaching, presence of change champion, devolution and political good-will. Four sustainability inhibitors identified were: human resources constraints, policy implementation, misalignment of projects with daily operations, devolution and political interference.

Conclusions The sustainability of institutional improvement strategies such as projects implemented post-leadership training in public and private health facilities depends on the quality of board members, communication management and institutionalisation of coaching culture. These findings are pertinent for planning and implementing similar health systems strengthening intervention in low-income countries.
Strengths and limitations of this study

- We ensured a cross-sectional representation of viewpoints from different healthcare contexts by incorporating health managers' views from both public and private facilities (for-profit and not for profit).

- The utilisation of in-depth interviews elicited rich data about context-specific challenges influencing the sustainability of institutional improvement projects addressed.

- The programme evaluation was undertaken by a team from the same institution, which was part of the programme implementation consortium; hence, measures had to be put in place during data collection and analysis to mitigate the potential conflict of interest.

\section{BACKGROUND}

Implementing a sustainable health system performance intervention is a persisting challenge endured by all healthcare leaders and their stakeholders. ${ }^{1}$ Building strong and sustainable health systems, therefore, require innovation, including innovative education for health workers. ${ }^{2}$ Examples of such innovations are the universal and contextspecific health reforms. For example, in Kenya, health service delivery was devolved from the national government to the counties in $2013 .^{3}$ Evidence suggests that decentralisation improves efficiency, equity, access, delivery, accountability and responsiveness of the health system. ${ }^{4}$ However, reports indicate that health system performance in Kenya remains poor. ${ }^{5}$ The main issue is poor leadership resulting in poor health system performance (inefficient use of available resources and underfunding of critical programmes) ${ }^{6}$ However, most training in countries such as Kenya focuses on 'leaders' (individual) development as opposed to 'leadership' training (....as opposed to 'leadership' training which encompased the development of a group 
from an organisation). ${ }^{7}$ Even with the widely implemented innovative health reforms, the sustainability of such innovations is deemed successful at initiation and fails to become part of an organisational routine or scale-up. This is often blamed on weak health systems. ${ }^{289}$

Sustainability is described as the final stage of programme use in which the programme is incorporated into organisational routines so that it is maintained once the initial programme funding, adopters or programme champion are no longer present. ${ }^{10}$ Programme sustainability is renowned as a vital component of any successful project and therefore spurs investment in educational improvement to ensure the sustainability of stiff-won gains. ${ }^{9}$ The expanding body of literature synthesis by Shediac-Rizkallah and Bone defined sustainability as 'the general phenomenon of program continuation'. ${ }^{11}$ There is rising recognition that the level to which new programmes are sustained is influenced by several diverse factors, and demands more insights on what and how these factors interact. ${ }^{12}$ The common emerging themes on factors affecting programme sustainability include: (a) the design of the programme, (b) available resources, (c) existing organisational structure, (d) availability of powerful change champions, (e) evidence-based output and (f) continuous improvement using data. ${ }^{13-15}$ In a more recent systematic review by Iwelunmor and colleagues on the sustainability of health interventions implemented in sub-Saharan Africa, community ownership and mobilisation were recognised as crucial facilitators for intervention sustainability. At the same time, the social, ecological conditions, as well as societal upheavals, were cited as barriers that influenced the sustainment of interventions in sub-Saharan Africa. ${ }^{16}$ Even with this evidence, little is known about the context-specific sustainability enablers and barriers and how they interact with each other in different health sectors. Research evidence illustrates that a lot can be enhanced merely by paying attention to how health providers manage and lead their facilities and teams. ${ }^{17-19}$

Although the implementation of new knowledge has been a major research interest area for many scholars with interest in understanding the transfer of the training process, sustainability remains the main concern for many programmes. ${ }^{20}$ Regrettably, sustainability is barely incorporated in the many programmes, and the existing evaluation processes are designed to assess the immediate programme outcome and neglect long-term sustainability. ${ }^{21}$ Pluye $e t a l$ s study findings suggest that to ensure the continuation of a successful pilot programme, the change process and the concept of sustainability need to be elaborated beyond theories to practical application. ${ }^{22}$ Scholars have developed an overarching multilevel framework on influences on sustainability, including intervention characteristics, organisational and community environment (Stirman et al 2018). ${ }^{12}$ A recent study on the sustainability of evidence-based intervention by Shelton $e t a l^{15}$ posit that even though the earlier literature on sustainability-focused largely on routinisation of a new set of practices into organisations' routine operation; the used frameworks did not fully address sustainability in the context of change over time. From the methodological point of view, Wiltsey Stirman and colleagues, ${ }^{, 12}$ studies reported that most sustainability studies had used self-reporting, with few studies using fidelity assessment. These are the research gaps that the current study seeks to address from the context of Kenya's devolved health system.

Our study was guided by the Iwelunmor and colleagues' proposed comprehensive conceptual sustainability framework that broadly maps the terrain of findings from interventions implemented in sub-Saharan Africa. ${ }^{16}$ The framework emphasises the role of organisational factors in influencing sustainability, which is the core component embedded within the overall life cycle of an intervention that evolves through time. ${ }^{16}$

\section{Intervention setting}

Our study is based on ongoing health managers' training 'The Leading High-performing Healthcare Organisations' (LeHHO). The programme was developed and implemented in the year 2010 by Strathmore Business School (SBS) in partnership with Management Sciences for Health and Ministry of Health under the funding support by the US Agency for International Development (USAID). The leadership programme aims to enable senior national and county management teams to address the most critical health system challenges in a devolved system of government. The programme participants comprised heads of departments and programmes from both the public and private facilities (for-profit and not for profit). The programme has been implemented in six cohort cycles between the years (2010-2016) trained 165 leaders. The programme cohort cycle refers to a team of jointly trained health managers within a 9-month period. A cohort cycle is composed of: five workshop modules, four-team coaching sessions and one cross-learning site visit. Each workshop module is equivalent to four classroom days, and a coaching session takes between 60 and $120 \mathrm{~min}$. A critical part of the training is the incorporation of institutional improvement projects which have to be undertaken by teams from the participating institution. The programme training approach is informed by the managing for results framework by the Management Sciences of Health, to gain insights on health system performance indicators changes. ${ }^{23}$ Even though the leadership development training was funded by the USAID, the implementation of the projects during and after the training was based on how the participants could innovatively use the existing resource to create a positive change. The idea is that it is through the implementation of such projects that participants are able to translate leadership training theory into practice and have a positive impact on institutional health system performance. The leadership outcome, therefore, depends on the desired results selected by the project teams and their specific context. The projects selected were informed by either county 
or institutional strategic plans, and across the six pillars of the health system. ${ }^{24}$ Some of the possible contextspecific intermediate outcomes included changes in work climate or management systems and processes such as: (a) finance, (b) human resources and (c) information management and research. The anticipated evaluation of long-term outcomes included service delivery results such as improved quality of care, increased access, and utilisation of services, or better client satisfaction, and lower operational costs.

This study covers the period from 2010 to 2016. During this period, 69 such projects have been undertaken by LeHHO trainees drawn from 39 health facilities in 19 counties in Kenya. The design of the leadership training programme was informed by the rationale that its success and sustainability would depend: (a) working with key stakeholders in health with the intention of addressing the devolved health system challenges, (b) integrating postclassroom training team-coaching modules around institutional improvement projects, which is a newly adapted concept in Kenya and most part of Africa, (c) designing the training programme to suit all the health sector needs (public, private and the faith-based health facilities and (d) planning the training programme with a deliberate consideration on how monitoring and evaluation process could be implemented throughout the programme. Most health leadership training programmes in Kenya are never evaluated for their impact and sustainability. To the best of our knowledge, LeHHO is the first qualitative study on the sustainability of health systems improvement intervention in the context of leadership development training in Kenya, post-devolution.

Although LeHHO programme shares similarities with the majority of training programmes whereby outcomes are measured during or just after the training programme on learning and retention of learnt knowledge. In contrast, studies on the application of the newly acquired knowledge through training on real workplace challenges demonstrate that the training's effect should be evaluated by measuring how trained skills have been maintained and generalised by the trainee after being on the job for some time..$^{25}$ The most recent and comparable leadership development case studies on the impact of leadership on organisational performance ${ }^{17} 19$ 26-28; reported a positive effect of the leadership development training on the selected health indicators. These studies further demonstrated that there was the sustainability of the positive results beyond the training. However, the understanding of how the transferred knowledge in the form of projects is sustained in the work environment is warranted. Additionally, all the highlighted studies were undertaken in public sector facilities hence limiting the findings to the public sector only. Another research gap is that the study participants and programme targets were front line health service providers, and hence generalisation of the findings is explicitly limited to service delivery health workers. Our current study builds on our earlier qualitative study on knowledge transfer enablers and barriers in project-based experiential learning in healthcare organisations in Kenya by Chelagat and colleagues. ${ }^{29}$ In our study, we reported: training design; work environment climate, trainee characteristics; team-based coaching; and leveraging on occurring opportunities as the key enablers of knowledge transfer within the current study participants' work context.

Additionally, the same study reported: lack of management support, human resources constraint (capacity and qualification), misalignment to the board's priorities, industrial action by frontline health services personnel (nurses and doctors), negative, poor prioritisation and poor communicate of a shared vision as the key transfer barriers. ${ }^{29}$ For this study, 'knowledge transfer' was described as 'the extent of successfully implemented priority projects and realised goals' to improve health systems performance in different counties in Kenya. 'Sustainability', on the other hand, was described as 'the extent to which institutional project teams maintained the positively attained project results with 24-60 months post the leadership training'. We focused on the action of implementation of leadership development practices through a continuous application of leadership and management practices to address real workplace challenges as a team. The projects provided practical relevance and most pertinent effectiveness measures concerning the training curriculum content and transfer design.

Our study aimed to investigate the health managers perceived sustainability status of the diverse health facilities institutional improvement projects and their experiences on factors that facilitated or constrained their maintenance, with intentions of informing relevant strategies or policies in Kenya's health sector. In this study, we make three key contributions towards these objectives. First, we described the sustainability status of the successfully implemented projects. Second, we explored, summarise and present context-specific sustainability transfer facilitators and constraints in diverse health systems settings. Third, we highlight the lessons learnt and policy implications for the national, county and longterm organisational plans for the innovative learning implementations in low-resource settings. The study, therefore, sought to answer the following research questions: (a) what is the sustainability status of the 33 successfully implemented team projects within 24-60 months post leadership development training? (b) what factors facilitated or constrained the sustainability of the implemented priority project indicators post-training at the workplace? (c) what are the lessons learnt and policy implications for the national, county and organisational long-term sustainability plans in low-resource settings?

\section{METHODS}

This qualitative substudy was nested within a quasiexperimental study on the 'assessment of the impact of leadership training on health system performance in 
selected counties in Kenya'. Among the issues examined in the larger study were leadership priority challenges at the health facility level, implementation status of priority challenge projects, factors influencing project implementation at the workplace and impact of the institutional improvement projects on the selected outcome indicators. The 33 respondents who had successfully implemented their team projects constituted a subsample of 39 respondents in the larger study and participated in the study. We used a convenience sample approach, 33 facility and senior managers were contacted by email with an interview request. These were managers who had participated in the LeHHO training programme. The study participants were identified through the training institutional projects team reports. The case health facilities comprised: 23 public, 10 faith-based and 6 private identified through the LeHHO programme 2011-2016 programme. Three criteria informed the case project-teams selection. First, we ensured that the teams were a good representation for the public, private and faith-based health facilities within the 19 counties in Kenya. Second, the teams had successfully implemented the selected priority project at the end of the training. Third, there was at least one or more trained team members' still working in the same organisation post-training. Within each of the facility teams, the units of analysis forming the basis of data gathering were the team's implemented project and the study respondents were the project team leaders or project team representatives. For this study, 33 respondents from the (20 public, 9 faith-based and 4 private) health facilities who had successfully implemented their institutional improvement projects, as reported by Chelagat and colleagues, participated in the study. ${ }^{29}$ The results of the study revealed that the implementation success rate was $85 \%{ }^{29}$ This implies that 33 out of the 39 projects prioritised were successfully implemented as a result of LeHHO training output.

The proxy measure of the success of the leadership training was the completion of the implementation of the institutional improvement project as per the agreed team's action plan. The project activities and the desired measurable result were agreed on at the beginning of the training. Based on the preceding study by Chelagat $e t a{ }^{29}$ the end-line data were measured against the baseline and reported at the experience sharing workshop, marking the end of the 9-month leadership training and the end of any institutional support such as coaching from SBS. A project was considered sustained if the project indicators presented at the end of the 9-month period are maintained or improved within the 24-60 months period post-training. A summary of the 39 projects and their indicators are presented in online supplemental file 1. All the project-team leaders and representatives were sent letters via email as an initial invitation to participate in the study. Follow-up telephone calls were made to confirm participation and book interview appointments for those who had expressed willingness to contribute to the study.
Interviews were conducted using a semi-structured interview guide between August and December 2018.

\section{Data collection}

In-depth interviews were conducted to discuss the health managers perceived status of their institutional improvement project implemented beyond the 24 months of leadership training. In-depth interviews contributed to a profound understanding of participant's perspectives and the relationship between the sustainability of the results and the contextual factors that could have influenced the sustainability of the institutional performance improvement initiative. Sustainability occurs when the positive implementation results are maintained 24-60 months post-training. A qualitative design was considered ideal for studying sustainability outcomes because in-depth interviews provide insight into why some projects were sustained while others were not, and promote understanding of differential processes occurring across context. $^{30}$

We structured the guide (online supplemental file 2) for key informant interviews starting with general introductory questions. The interview guide was pilot-tested through cognitive interviewing, as suggested by Collins and revised as needed prior to the study. ${ }^{31}$ Participants were asked about their role and experiences in implementing maintaining the priority challenge projects into their facility operation and their opinion on sustainability drivers and inhibitors in their context. The interviewers explored additional information on how these factors could be reinforced or mitigated for better results. We specifically focused on their experiences during project implementation, which consequently presented opportunities for immediate knowledge application and linked class with the work environment challenges. In total, 33 in-depth face-to-face interviews were conducted by the research assistant Anne Muisyo (AM) and the principal investigator Tecla Chelagat (TC) at the respondent's health facilities. To ensure consistency and mitigate potential bias by the principal investigator due to affiliation with the programme implementing institution, the pilot exercise was jointly done by the principal investigator (TC) and the research assistant (AM). Additionally, from the total of 33 face-to-face interviews were conducted in this study, only 2 interviews were done jointly by the principal investigator and the research assistance, while the remaining 31 interviews were singly undertaken by the research assistant. The selection of the research assistant was not only informed by her technical expertise, but also, we ensured she had no prior knowledge of the programme or interaction with the programme alumni. Interviews were conducted in English and each interview lasted between 45 and $60 \mathrm{~min}$. The interviews were recorded using portable recorders, and supplementary notes were taken during the interview. ${ }^{32}$ Daily debrief was done by AM and TC to monitor any emerging issues or concerns that needed urgent attention. 


\section{Data analysis}

Data analysis was conducted by the first author (TC) and the second author (GK). The recorded interviews were then transcribed verbatim and verified for accuracy. The transcripts were then cross-checked with the field notes for consistency. The transcripts were clustered to public, private and faith-based facilities and then analysed separately per sector. Analysis began with open coding to understand sustainability drivers and barriers from health managers' perspectives using the NVivo V.11 software. ${ }^{33}$ Each transcript was then read independently and the emerging codes and themes were analysed by the coauthors. The emerging themes were subsequently analysed against the sustainability of health innovations literature, and new emerging supplementary themes were also discerned, culminating in thematic analysis. To ease comparison, matrices of all identified codes were generated among the health managers, project type and across the health sectors. Illustrative quotes representing a range of health manager's views were highlighted to elucidate each theme for reporting. Health managers also facilitated the dissemination of research findings through alumni breakfast forums and institutional breakfast series. Power-point slides were shared with respective institutions through the programme alumni. The study findings are presented according to O'Brien and colleagues' Standards for Reporting Qualitative Research (SRQR) guidance for reporting qualitative research. ${ }^{34}$

The participants were informed about voluntary participation. They were assured anonymity and that their names and identities are kept confidential. To ensure security, all the printed research materials were kept in a locked room within the health facilities and training institution. The leadership knowledge transfer data were not considered as personal data since the study did not involve human biological material.

\section{Indirect patient and public involvement}

This study was done without direct patients and public involvement in the designing of the research questions and the overall implementation of the study.

\section{RESULTS}

The study findings are presented in five subsections: (a) demographic characteristics of participants, (b) the sustainability status of the successfully implemented projects, (c) factors that facilitated and constrained sustainability of the implemented institutional improvement projects indicators post-training, (d) the sustainability drivers for the implemented institutional improvement projects per health sector and (e) factors that constrained the sustainability of the implemented priority projects indicators post-training at the workplace.

\section{Demographic characteristics of participants}

From the 33 purposely selected team-based projects, a sum of $(n=20) 67 \%$ were from public health, $(n=9)$

\begin{tabular}{lll}
\hline Table 1 & \multicolumn{2}{l}{ Participants demographic information } \\
\hline Item & Category & Frequency (n) and (\%) \\
\hline Health facility & Public/government & $20(67 \%)$ \\
type & $\begin{array}{l}\text { Faith-based } \\
\text { (private-not for }\end{array}$ & $9(27 \%)$ \\
& profit) & \\
& Private (for profit) & $4(12 \%)$ \\
Gender & Male & $12(36 \%)$ \\
& Female & $21(64 \%)$ \\
Age category & 26-35years & $4(12 \%)$ \\
& 36-45years & $11(33 \%)$ \\
& 46-55years & $15(45 \%)$ \\
& $>55 y e a r s$ & $3(9 \%)$ \\
Highest & Bachelor degree & $11(33 \%)$ \\
education level & Master degree & $19(58 \%)$ \\
& Doctoral degree & $1(3 \%)$ \\
& Others & $2(6 \%)$ \\
\hline
\end{tabular}

$27 \%$ from the faith-based and, $(n=4) 12 \%$ were from the private health facilities. The study participants comprised $21(64 \%)$ women and $12(36 \%)$ men. Nearly half $(45 \%)$ of the participants were between the age category of 46 and 55 years. A total of $58 \%$ of the participants had a master's degree. Table 1 provides a summary of the study participant's characteristics.

\section{Sustainability status of the successfully implemented projects}

Two broad contextual categories of project sustainability rates are reported in this study; projects that were sustained post leadership training and those that were not sustained. The sustainability status of the 33 successfully implemented team projects was $85 \%$. Out of the 20 successfully implemented projects for public health facilities, 15 were sustained for a period of 24-60 months. One of the nine successfully implemented projects for the faith-based health facilities failed to sustain. Interestingly, all the projects implemented for the private health facility teams were sustained. The sustainability variances in the different contexts reflect the varying needs and concerns in the different health facilities.

Factors that facilitated and constrained the sustainability of the implemented institutional improvement projects indicators post-training.

Seven main themes that illustrate sustainability key drivers emerged: programme design, stakeholder's buy-in, quality of the board members, communication management, the institutionalisation of a coaching culture, presence of the change champion, devolution and political good-will. While LeHHO programme design was customised to Kenya's health systems' needs through stakeholder alignment meetings, there were sector-specific drivers that were experienced in one sector as compared with others. The quality of board members, communication management and institutionalisation of coaching culture was the 
Table 2 The number one key enabler and barrier to the sustainment of the project's positive results as identified by different project teams

\begin{tabular}{|c|c|c|}
\hline Institution type & Key enablers to sustainability & Key barriers to sustainability \\
\hline $\begin{array}{l}\text { Public } \\
(\mathrm{n}=20)\end{array}$ & 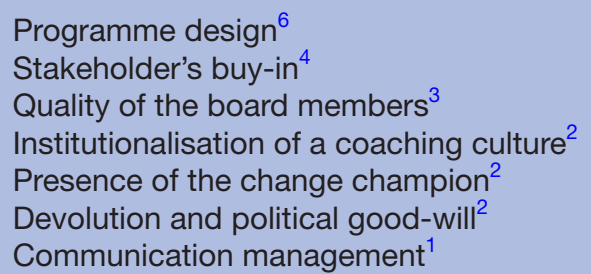 & $\begin{array}{l}\text { Devolution and political interference } \\
\text { Human resource shortage } \\
\text { Misalignment of the project goals to } \\
\text { teams' day to day operations }{ }^{1}\end{array}$ \\
\hline $\begin{array}{l}\text { Private } \\
(n=5)\end{array}$ & $\begin{array}{l}\text { Stakeholder's buy-in }{ }^{3} \\
\text { Communication management }\end{array}$ & \\
\hline
\end{tabular}

significant cross-cutting drivers' related sustainability of project results in all the health sectors; programme design was highly rated as a key sustainability driver for the public health facilities, stakeholder-buy for the faith-based and private health facilities. Overall, stakeholder's buy-in was the most mentioned cross-cutting enabler in all the health sectors. Even though communication rated low as the first key driver identified by the mangers, further analysis shows that it was the most mentioned (90\%) across the sectors. Human resource shortage and attrition were mentioned as the number one key inhibitor of sustainability across the sectors. It is also worth noting that devolution and political interference were mentioned as the key drivers and inhibitors among the public health facilities only. Table 2 presents the enablers and barriers identified as the main influence on sustainability.

The themes and subthemes are further categorised into both universally and sector-specific recognise factors that facilitated the sustenance of institutional priority project post-LeHHO training. These results are in general agreement with the common emerging themes reported earlier by scholars such as, ${ }^{13}$ which include: design of the programme, available resources, existing organisational structure, availability of powerful change champions, evidence-based output and continuous improvement using data. From the context perspective, four unique themes emerged as a sustainability enabler at the workplace: (a) quality of board members, (b) institutionalisation of coaching culture, (c) communication management and (d) devolution and political good-will. Further analysis led to the emergence of new subthemes for each driver identified by context. Table 3 summarises the essence of the perceived sustainability drivers with respect to the health sector context.

These themes suggested why some projects were thriving, and are further described with quotations from healthcare managers to illustrate each subtheme. The emerging themes and subthemes underscore the contextspecific fundamental elements deemed necessary for the sustainability of knowledge transfer.

\section{Driver 1: programme design}

The programme design nature of LeHHO programme was widely recognised by the interviewees as a key enabler in all health sectors. The build-in knowledge sustainability approach, such as; sector need-driven curriculum, selective team recruitment, challenge-based driven learning blended with team coaching, was highlighted as key enablers. Using the words of senior Ministry of Health manager: 'the programme was codesigned by different key healthcare industry players with different health sector challenges in mind'. The description was accentuated by other interviewees:

... initially, I thought the program was designed for public health sector only; however utilization of case studies and challenge model approach enabled us to speak to each other across health sectors and we realized that we are struggling with similar problems and collaboration was the only way out ... the program enabled us to sit in one room and facilitated debates such as 'Kenya's Health Agenda Initiative' which was initiated by program alumni .... (Health manager at a private facility 05)

... the senior management were part of the training program hence it was easy to influence the rest of the staff to implement the project ... our institution also has an established systematic way of handling challenges such as record department, this made our implementation and sustainability of project indicators seamless .... (Health manager at a public health facility 017)

The findings revealed that leadership development through teams was recognised as a positive approach in capacity building of the human resources for health.

\section{Driver 2: stakeholder's buy-in}

In some contexts, stakeholder buy-in resulted in a positive work climate for a successful change process aligned to the achievement of institutional strategic plan, vision and mission. Some interviewees noted that their ability 
Table 3 Key sustainability drivers for the implemented priority projects per health sector

\begin{tabular}{|c|c|c|c|}
\hline Public health facility & Public health facility & Faith-based health facility & Private health facility \\
\hline \multicolumn{4}{|l|}{ Programme design } \\
\hline Rigorous recruitment & $\sqrt{ }$ & $\sqrt{ }$ & \\
\hline Team recruitment & $\sqrt{ }$ & $\sqrt{ }$ & \\
\hline Programme curriculum & $\sqrt{ }$ & $\sqrt{ }$ & $\sqrt{ }$ \\
\hline \multicolumn{4}{|l|}{ Stakeholder's buy-in } \\
\hline Ministry of health and county government & $\sqrt{ }$ & & \\
\hline Senior management & $\sqrt{ }$ & $\sqrt{ }$ & $\sqrt{ }$ \\
\hline Other key stakeholders & $\sqrt{ }$ & & \\
\hline \multicolumn{4}{|l|}{ Quality of board members } \\
\hline $\begin{array}{l}\text { Alignment to board members strategic } \\
\text { objectives }\end{array}$ & $\sqrt{ }$ & $\sqrt{ }$ & $\sqrt{ }$ \\
\hline Good governance & $\sqrt{ }$ & $\sqrt{ }$ & $\sqrt{ }$ \\
\hline Competent and trained board & $\sqrt{ }$ & $\sqrt{ }$ & $\sqrt{ }$ \\
\hline \multicolumn{4}{|l|}{ Communication management } \\
\hline Top management communication & $\sqrt{ }$ & $\sqrt{ }$ & $\sqrt{ }$ \\
\hline Team communication & $\sqrt{ }$ & $\sqrt{ }$ & $\sqrt{ }$ \\
\hline Social Media & $\sqrt{ }$ & & \\
\hline \multicolumn{4}{|l|}{ Institutionalisation of a coaching culture } \\
\hline Coaching chain & $\sqrt{ }$ & $\sqrt{ }$ & $\sqrt{ }$ \\
\hline Scaling-up of project goals & $\sqrt{ }$ & $\sqrt{ }$ & $\sqrt{ }$ \\
\hline Communication and leadership tool & $\sqrt{ }$ & $\sqrt{ }$ & $\sqrt{ }$ \\
\hline \multicolumn{4}{|l|}{ Presence of the change champion } \\
\hline Vision bearers & $\sqrt{ }$ & $\sqrt{ }$ & $\sqrt{ }$ \\
\hline Change agents retention & $\sqrt{ }$ & $\sqrt{ }$ & \\
\hline \multicolumn{4}{|l|}{ Devolution and political good-will } \\
\hline Accountability & $\sqrt{ }$ & & \\
\hline Decentralised power & $\sqrt{ }$ & & \\
\hline Resource mobilisation & $\sqrt{ }$ & $\sqrt{ }$ & \\
\hline Collaborations through partnerships & $\sqrt{ }$ & $\sqrt{ }$ & $\sqrt{ }$ \\
\hline
\end{tabular}

to influence key stakeholders to support their prioritised catalyst project during and after the training was a great enabler for transfer and maintenance of positive gains resulting from the implementation of the projects:

Our recruitment to the program was done from the top management level even though we had not expressed interest for the training ... after the first module we realized why we were chosen to attend the training and not delivering the expected results was not an option ... we had to do a lot of lobbying at all levels in the organization to ensure that all key stakeholders involved in our chosen projects were on board and willing to support us to not only implement but scale-up the project after the training ... we nominated the most resistant team members to be project leads and we kept updating the top-management on the progress, challenges and support need throughout the implementation and scaling-up phase .... (Health manager at a private facility 04)

... we were advised to choose projects which we can implement with the limited available resources available but with high impact ... we mobilized senior management support, community health workers and health partners for support ... we noted that it is not possible to reach out to pregnant mothers in the village without the involvement of the community health worker ... we were lucky to get donors to pay stepped for the community health workers, we are able to sustain the results after the buy-in from county government to support community health workers stipends. (Health manager at a public health facility 05) 
The finding that team training across different levels of organisational leadership was an apparent key enabler of sustainability in all contexts of health provision.

\section{Driver 3: communication management}

Over $90 \%$ of the interviewees perceived their transformed communication capacity enabled them to start looking at each from different lenses. They reported that their main breakthrough during the training was the creation of a positive work climate that was easily attainable through effective communication and appreciation of each staff member as a team. Remarkable, the use of social media was highlighted as a key game changer for accountability in the space of health service delivery as demonstrated in the following quotes:

During our experience sharing workshop in the LeHHO module, our biggest was, 'what did we know before?' The greatest eye-opener was learning the art of effective communication ... we discovered that given that we are working in a challenging environment, less jovial and with patients whose patience is tested constantly, the only way to create a positive environment for both staff and patients was adapting effective communication skills and emotional intelligence ... these are competencies not trained in the medical school yet very crucial especially when managing and leading health facilities in a resource-scarce environment. (Health manager at a public health facility 016)

... use of social media as a communication platform has transformed our level of performance for the better ... before social media, many malpractices and poor quality of health services provision were never reported and no one was held accountable ......

(Health manager at a faith-based facility 04 )

The respondent's report suggests that effective communication management is a key enabler of a positive work environment that, in turn, supports thriving initiatives through team cohesion towards a shared vision.

\section{Driver 4: quality of board members}

The ability of board members to add value to the existing organisational resources through inspired leadership, sound management and good stewardship, was highlighted as a key enabler. At the board level, there was a perception that focusing on training the board members first before the rest of the managers resulted in a competent, and aligned board, leading to effective knowledge transfer and generalisation at the workplace and improved health facility governance:

Two of the team members were board members this was a great plus to our team since they understood the program demands and expectations which resulted to good governance and availability of necessary resources to sustain projects positive results ... through the boards' support we were able to enroll more managers to the LeHHO training hence more positive outcomes at the organizational level. (Health manager at a faith-based facility 01)

There was a widespread consensus among the health managers who cited that building the board's capacity on leadership and governance was a key enabler in ensuring that the institutional improvement projects are not only implemented but sustained.

\section{Driver 5: institutionalisation of a coaching culture}

Several interviewees perceived the support on the institutionalisation of a coaching culture, especially among the line managers as a key recipe for the continuance of positive results achieved as a result of project implementation. Embedding coaching as a leadership and communication tool generated a pool of coaches at workplace resulting in a strong and consistent coaching chain for the sustenance of the project goals within different departments:

In addition to leadership and management skills acquired in LeHHO class, team coaching introduced to our board members during the training created a unified vision; consequently they were able to influence other management and staffs to contribute to the vision of the project .... (Health manager at a public health facility 08)

The tangible impact achieved from implementation of catalyst projects inspired the management to seek support from the Strathmore coaches to offer institutional support with the aim of institutionalization of coaching culture through training of departmental managers ... we have scaled-up coaching practice as a leadership development approach across all levels in the organization ... we avoid telling staff what to but instead we challenge, support and provide them with feedback ... we have grown in 3 folds ... our participation in the training started when we had one facility only, now we have opened nine branches across the country. (Health manager at a private facility 05 )

\section{Driver 6: the presence of the change champion}

Several interviewees reported that despite the fastemerging promotions opportunities for managers after LeHHO training, institutions who promoted and retained their managers sustained their results better than those with a high attrition rate of the transformation vision bearers:

... our organization had invested heavily in the capacity building of the management team and heads of departments ... we, therefore, had a pool of LeHHO alumni within our organization; hence it catalyzed implementation of priority challenge projects in different departments contributing to overall organizational performance improvement. (Health manager at a faith-based facility 04)

Team recruitment to the leadership training was suggested as an effective strategy in ensuring that the 
trained managers (change champions) are retained in the same institution in the case of transfers or retirements, which was cited as a common attrition factor in Kenya's health sector.

\section{Driver 7: devolution and political good-will}

There was a consistent reporting from the public, private and faith-based health facilities managers on the impact of devolution of health systems to the 47 counties even though more impact was felt in the public health facilities. Critical health service provision issues such as accountability, decentralised power, resource mobilisation and collaborations through partnerships were cited as key by-products of devolution of the health services:

Devolution cascaded decision making and services closer to the people; therefore, health management teams are able to reach out to the county management team for support and this worked (...). Devolution works well also for patients in that they are now, only a phone call away from airing their grievances to the county governor whenever their healthcare needs are not met ... this kind of accountability has stretched us to be consistent with our health service provision. (Health manager at a public health facility 04)

Political good-will facilitated our project progress, being at governance position, we could access the resource, influence buy-in, and support from hospital management ... we motivated nurses, maternity $\&$ antenatal department staff and also we successfully mobilized community through community health workers' engagement. (Health manager at a public health facility 012)

The effect of devolution on the implementation and sustainability of institutional improvement projects were largely cross-sectoral because devolution promoted crosssector partnership in different counties in Kenya.

Factors that constrained the sustainability of the implemented priority projects indicators post-training at the workplace

In this section, we address the question, 'Under what conditions were the catalyst projects positive results unsustainable?'. Four key themes emerged as common to healthcare managers from the public and faith-based health facilities' workplace experiences on factors that hindered maintenance of the catalyst projects results over time: (a) human resource constraint; (b) inability to translate policy from paper to people; (c) misalignment of project goals with teams' day to day operations; (d) devolution and political interference. We describe these key themes with a commendable excerpt from healthcare managers to point-up each theme.

\section{Inhibitor 1: human resource constraints}

Despite the significant emphasis on human resource capacity building through training as a resolution to human resource challenges, constraints to effective strategies, human resource retention are many. Our study participants emphasised the inadequate human resources for health as a binding constraint to the improvement of health systems performance, especially in the current devolved health system in Kenya. The comment was evidently reflected by a couple of exciting management systems improvement projects such as automation of hospital patient and procurement systems, which are now performing at $50 \%$ capacity and in some departments $0 \%$ due to high staff turnover. It was evident that some projects which were not sustained as a result of: championing team members, information technology team or nurses exiting the institutions. These sentiments were common only in public and faith-based health facilities. Our participant noted the following:

... the project target indicators on improving customer satisfaction in the outpatient and inpatient department were successfully achieved by the end of the training session ... however, customer satisfaction rates declined steadily ... tracing back we attributed the degeneration to lose of change champions ... all the three trained managers left the institution within the three years after the training .... (Health manager at a public health facility 020 )

... we accepted change on the improvement of services ... my team worked together as change agents and we co-opted a non-trained manager to be the project manager so that we can transfer the ownership to key departments ... it worked well for us because even with some team members leaving the institution, our project was not significantly impacted ... despite the positive changes, our performance plummeted as a result of 'mass-exodus; of frontline workers especially nurses ... we do all the recruitment and training and before we could 'reap the fruits' ... they move to private or big public hospitals .... (Health manager at a faith-based facility 07 )

There was widespread reporting on the effect of human resource constraints on the sustenance of the positive results from the implemented project, especially for the public and faith-based health facilities.

\section{Inhibitor 2: inability to translate policy from paper to people}

Even though the inability to translate policy from paper to people was not mentioned as the first key barrier, one facility manager participants perceived that investing in getting a full board members buy-in and support from the initiation of the project would be beneficial in cultivating sustainability culture for all projects implemented:

... our project involved development and operationalization of human resource policy manual, and we were able to achieve the project's first phase of implementation which was to develop an approved policy manual ... however, our project stalled and our manual was never operationalized to-date, but was shelved until unknown time ... one of the key reasons for the delay was that the phase of the project was not part of 
the board members' priorities .... (Health manager at a faith-based facility 010 )

These findings relate to the earlier mentioned enabler (quality of board members), in that without inspired leadership and transparent governance, it is not possible to sustain the health systems improvement initiatives.

\section{Inhibitor 3: misalignment of the project goals to teams' day to day operations}

The perceived importance of application of leadership practices at the work environment in the case of this study was sufficient to inspire change agents to identify and initiate the implementation of the catalyst project but not adequate to sustain within the institutional existing structure and operations. This could be attributed to the intense resources requirements to sustain the projects. It is common in many healthcare institutions where practicing doctors are part of the hospital management team. Hence, implementation of leadership and management project is possible as long as the trained managers are working under the reduced clinical workload. However, when the training comes to an end that the project is fully integrated into the organisation, the sustainability of results quickly declines due to change in priorities:

... I am a practicing pediatrician and I attended the training alone. I championed a project on quality improvement and accreditation for the whole hospital ... it was a great struggle. Still, I was able to mobilize a team and successfully implemented the project ... my concern is that I am back to my full workload. I see my patients throughout my working hour ... the anticipated implementation phases of our project have stalled because my day to day work is not directly aligned to quality improvement department .... (Health manager at a private health facility 01 )

\section{Inhibitor 4: devolution and political interference}

Even though devolution was earlier cited as a key enabler of project sustainability, some teams contrasted this perception. The devolved health system encouraged a lot of the health workforce transfers from one county to another, therefore creating gaps in some facilities. Additionally, the first phase of devolution ensured that all necessary resources were prioritised and timely, however many counties were not able to sustain the promise hence resulting in a decline in performance:

... our team had one major enabler that led to the successful implementation of our institutional improvement project, 'devolution of the health systems' ... devolved funds from the central government to county-level made it easy to access adequate resources to carry out projects .... (Health manager at a public facility 03 )

A couple of interviewee from both the public and private sectors cited politics at the County level as a key deterrent to the sustainability of projects:
... every newly elected political leader takes over leadership with a bag of promises to fulfill to their voters ... this positive gesture comes with misdirection of funds from the existing projects implemented by the outgoing politician because each leader is focusing on implementing their own promised projects without leveraging on the existing gains and milestones ... for example, our catalyst project was to ensure zero stock-out of essential medicines and we received support from the governor on the same; however, the positive results were not sustained since the new governor's priorities was expansion of infrastructure such as building new health facilities ... we, therefore, commissioned another project to align with the governers agenda. (Health manager at a public health facility 08 )

\section{DISCUSSION}

The objectives of this study were to investigate the health manager's perceptions on the status of their institutional improvement project and highlight specific institutional enablers and barriers to the sustainability of the successfully implemented project post leadership development training. The study further explored the contextual sustainability factors for the public and private health facilities in Kenya, with the intention of synthesising how different factors interact with each other in diverse health system contexts. The thematic construct which emerged suggest that sustenance of positive institutional improvement projects indicators across the health sectors are facilitated by seven important mechanisms: (a) programme design, (b) stakeholder's buy-in, (c) quality of the board members, (d) institutionalisation of a coaching culture, (e) presence of the change champion, (f) devolution and political good-will and $(\mathrm{g})$ communication management. Regardless of the health sector type, the findings suggest that even with a well-designed programme, stakeholder's buy-in is a critical factor to consider in all health institutions to ensure the sustainability of change initiatives. This is possible through sound stakeholder engagement and effective communication management. It takes good leadership and governance to promote a shared vision and inspiring work environment. ${ }^{19}$ These factors are linked with the creation of a positive work climate that enhances effective health workforce engagement and commitment despite the dynamic challenges and scarce resources. The findings principally reflect the perspective diverges from the growing body of literature on knowledge transfer sustenance about the interaction of sustainability elements and context. The synthesis of sustainability drivers using this approach places emphasis on how healthcare leaders can influence the work environment and the sustainable health workforce capacity building in different health system contexts. One of the unique contributions of this study is a glimpse into the effect of devolution of health services, the role of the 
board, politics and institutionalisation of a coaching culture in ensuring improved health service provision in Kenya.

The current study complements a recommendation by Shelton and colleagues who proposed the need to approach sustainability by focused not only on the routinisation of a new set of practices into organisations' routine operation but addressing sustainability in the context of change over time. ${ }^{15}$ This study further delineates the four fundamental sustainability inhibitors which were evident among the non-sustained project's outcome: (a) human resources constraints, (b) misalignment of project goals with teams' day to day operations, (c) inability to implement policies and (d) devolution of health services and negative politics. Most of these constraints were reported in the public health facilities and were attributed to the no-sustenance of the projects post the training period. These findings extend the work of, ${ }^{1315}$ which draws attention to the fact that even with pieces of evidence on factors affecting programme sustainability, a synthesis on how different sustainability enablers interact with each other in diverse health systems is deficient. This study further disaggregates the sustainability drivers into different health service delivery contexts in Kenya by examining how each theme and subthemes are represented in different health sectors (public, private and faith-based health facilities). The quality of board members, communication management and institutionalisation of coaching culture were the significant cross-cutting drivers' related sustainability of project results in all the health sectors.

An additional contribution of this study is the contextual exploratory examination of the proposed sustainability factors of on health systems strengthening intervention. ${ }^{13} 15$ Specifically, in the era of immense investment in the health system strengthening, new reforms such as the devolution of the health services and the felt influence of national and county politics, in driving health agenda in Kenya. Further than these simple associations, this study presents suggestions based on lessons learnt, on how the key stakeholders including policy makers can innovatively enhance sustainable generalisation of learnt knowledge and skills at work place such as: (a) constant application of leadership and management practices on the real workplace challenge which in turn leads sustainable health system performance improvement, (b) key stakeholder engagement for buy-in and support, (c) effective human resources engagement, (d) alignment of the training practices to the key stakeholders needs, (e) health organisations capacity building strategy should start with training of board members and team training to ensure inspired common vision at all levels, (f) an effective leadership programme should focus not only imparting knowledge but also inspire the heart and encourage application of knowledge through action and teamwork, (g) even though coaching is a relatively new leadership development concept, training institutions should build a compulsive evidence-based case that will convince partnering organisations why coaching should not only be embedded in leadership training but also within the transfer organisations and $(\mathrm{h})$ devolution of health services should be supported by strong sustainability policies such as resource mobilisation and taskshifting. Although there is a complex link devolution and political good-will as one of the sustainability drivers across the context, there is a single, strong connection between leadership development and community empowerment through joint decision making and ownership to enhance the culture of commitment, transparency and accountability by all. In fact, when leadership capacity building is done to target all the cadres in an organisation, the chances of sustaining the positive outcomes are very high than training the frontline workforce alone. By itself, the practice of team leadership development centred on the application of priority projects based on institutional projects is a crucial sustainability driver; this is because the approach creates and empowers more leaders inspired to face one challenge at a time. This implies that the training institutions in partnership with health service providers and other key stakeholders should design not only customised programmes for different staff cadres, but should focus on unifying the overall institutional agenda, which is the improvement in health service delivery performances.

\section{Strengths and limitation of this study}

In this section, we underscore the study's strengths and limitations in order to draw attention to opportunities for further research development. Anchored in a larger study and hence a potential series paper that builds on the previous study by Chelagat $e t a l,{ }^{29}$ the objective of the study was clearly stated. Additionally, this is the first qualitative inquiry study to explore the drivers and inhibitors of leadership development knowledge transfer sustainability in a healthcare context in sub-Saharan Africa. In addition to in-depth interviews for data collection. Despite the strengths and contributions of the current study, several potential limitations should be noted. First, the findings are based on data collected from a single training programme; thus, its interpretation and recommendations are applicable to the ongoing programme, but may not be as relevant to other leadership development interventions. Second, our study employed a qualitative design; hence, the findings on knowledge transfer enablers and barriers should be cautiously interpreted because they are based on the perceptions and views of health managers, excluding views of team members or institutional team members who cooperated in the project. Third, even though the study had, within the study comparison group, the study lacked a programme comparison group. Therefore, it is not possible explicitly to conclude that generalisation of knowledge occurred as a result of the implementation of leadership development practices alone. Fourth, the programme evaluation was undertaken by a team from the same institution, which was part of the programme implementation consortium; hence, measures had to be put in place during data collection and analysis to mitigate the potential conflict of 
interest. Fifth, although this study contributes to an understanding of issues that affect the sustainability of transferred knowledge at the work environment in the form of institutional improvement projects, good quality documentation of information on the scalability of these projects was unavailable in detail. Future studies might consider a more rigorous design with a comparison group.

\section{Implications of the study}

This study offers insight into the drivers of the sustainability of knowledge in the healthcare work environment. One of the significant implications for the implementation and sustenance of the project-based experiential learning is to enable Kenya's national and county health management teams to address the most critical health system challenges in a devolved health system of government. LeHHO programme was anchored on a powerful definition that 'leadership is enabling others to face challenges and achieve results in the complex situation'. ${ }^{23}$ The application of leadership practices ${ }^{17} 1928$ illustrating that even in a scarce resources setting, the practice of experiential leadership and embracing the notion that 'people can learn to lead and at any level by prioritising and taking on challenges to produce tangible results, is already a powerful antidote to low morale. ${ }^{28}$ This study also provides an additional contribution to sustainability research methodological rigour, which supports Wiltsey Stirman's and colleagues' study findings. They reported that most of the sustainability studies had used selfreporting, with few studies using fidelity assessment. ${ }^{12}$ From the policy perspective, the training institutions, healthcare service providers, funding agencies and the health policymakers should promote a sustainable health system leadership capacity building, which facilitates a positive work climate receptive to sustainable positive change. They should also focus on fostering positive politics that promote development hence improved health service delivery for all Kenyans, especially in the public health facilities.

\section{CONCLUSION}

This study explored health managers' perceived sustainability status, barriers and enablers of the institutional health system improvement projects within the period (24-60) months post leadership training. Based on the results for the team-based projects under study, we drew the following conclusion: (a) sustainability is not easily obtained if it is not embedded in the entire project cycle; but, when the programme sustainability is well thought through and embedded in the training curriculum co-owned by the training institution and the healthcare industry stakeholders, the probability of sustainability of training gains are very high; (b) even with an effective programme and sustainability plan, external factors such as political interference and human resources engagement warrant consideration and (c) transformational leadership, transparent politics, stakeholder communication and accountability can provide as special motivators for health facility managers to improve service delivery performance that are sustainable. This calls for continuous adaption of innovative problem-solving strategies in corresponding institutions through the integration of best practices into organisations' operations. This paper contributes to the nascent literature on knowledge transfer sustainability strategies.

Acknowledgements We are grateful to all the Health Managers and their facility teams from all participating counties in Kenya for sharing their experiences and access to their projects and data. We appreciate academic support from the Institute for Healthcare Management at Strathmore University PhD support group, specifically Ben Ngoye, Jackline Aridi, Mary Nyikuri and Eric Tama. We appreciate Annie Muisyo for her support in data collection.

Contributors TC was the principal investigator who conceptualised, designed, implemented, analysed and interpreted the data. GK reviewed the study objective, data analysis, interpretation of results and review of the manuscript. JR and J0 a significant contribution by providing advice on design, literature reviews, reviewed and suggested modification of drafts and approval of the final manuscript. The first author (TC) led in writing the manuscript and reviewed it based on comments from the other authors. All the authors reviewed and approved the submission of the final manuscript.

Funding The authors have not declared a specific grant for this research from any funding agency in the public, commercial or not-for-profit sectors.

Competing interests All the authors are affiliated with Strathmore University Business School. TC was part of the programme implementation team. GK, JR and $\mathrm{JO}$ participated in the evaluation of the programme, but they were not involved in the implementation phase.

\section{Patient consent for publication Not required.}

Ethics approval Ethical approval to conduct this study was obtained from the Strathmore University ethical review committees (Protocol ID No. SU-IRB 0243/18) and the permit to conduct the study in the counties was obtained from the National Commission for Science, Technology, and Innovation (NACOSTI/P/18/21001/23609). Written informed consent was obtained from the participants prior to their interview participation.

Provenance and peer review Not commissioned; externally peer reviewed.

Data availability statement Data are available in a public, open access repository. Data are available upon reasonable request. The data used for this study are qualitative, and the original transcripts can be made available from the first author upon reasonable request. Extra data can be accessed via the Dryad data repository at http://datadryad.org/ with the doi: 10.5061/dryad.gmsbcc2kd.

Supplemental material This content has been supplied by the author(s). It has not been vetted by BMJ Publishing Group Limited (BMJ) and may not have been peer-reviewed. Any opinions or recommendations discussed are solely those of the author(s) and are not endorsed by BMJ. BMJ disclaims all liability and responsibility arising from any reliance placed on the content. Where the content includes any translated material, BMJ does not warrant the accuracy and reliability of the translations (including but not limited to local regulations, clinical guidelines, terminology, drug names and drug dosages), and is not responsible for any error and/or omissions arising from translation and adaptation or otherwise.

Open access This is an open access article distributed in accordance with the Creative Commons Attribution Non Commercial (CC BY-NC 4.0) license, which permits others to distribute, remix, adapt, build upon this work non-commercially, and license their derivative works on different terms, provided the original work is properly cited, appropriate credit is given, any changes made indicated, and the use is non-commercial. See: http://creativecommons.org/licenses/by-nc/4.0/.

ORCID iD

Tecla Chelagat http://orcid.org/0000-0002-1312-8404

\section{REFERENCES}

1 Braithwaite J, Testa L, Lamprell G, et al. Built to last? the sustainability of health system improvements, interventions and 
change strategies: a study protocol for a systematic review. BMJ Open 2017;7:e018568-6.

2 World Health Organization. Key components of a well functioning health system. Geneva: World Health Organization, 2010.

3 Government of Kenya. The constitution of Kenya: 2010. Nairobi: Chief Registrar of the Judiciary, 2013: 26-30.

4 The World Bank. Decentralization in client countries: an evaluation of World bank support, 1990-2007. Washington, DC: The World Bank, 2008: 1990-2007.

5 KPMG. Devolution of healthcare services in Kenya: lessons learnt from other countries. KPMG services (proprietary) limited. KPMG Serv South Africa, 2013: 1-23.

6 Management Sciences for Health. Setting a shared strategic direction for health systems strengthening: series of guides for enhanced governance of the health sector and health institutions in low- and middle-income countries. 28. Cambridge, MA, 2014

7 Dopson S, Ferlie E, McGivern G. The impact of leadership and leadership development in higher education: a review of the literature and evidence. London, UK: Leadership Foundation for Higher Education, 2016.

8 World Health Organization. Designing and implementing training programs. Hum Resour Manag 2012;52.

9 Loman SL. Rodriguez BJ, Horner RH. Sustainability of a Targeted Intervention Package: First Step to Success in Oregon. $J$ Emot Behav Disord. 2010;18(3):178-91.

10 Kilbourne AM, Neumann MS, Pincus HA, et al. Implementing evidence-based interventions in health care: application of the replicating effective programs framework. Implementation Sci 2007:2:1-10.

11 Shediac-Rizkallah MC, Bone LR. Planning for the sustainability of community-based health programs. Health Educ Res 1998;13:87-108.

12 Wiltsey Stirman S, Kimberly J, Cook N, et al. The sustainability of new programs and innovations: a review of the empirical literature and recommendations for future research. Implement Sci 2012;7:17.

13 Gustafsson A, Nilsson L, Johnson MD. The role of quality practices in service organizations. Int $\mathrm{J}$ of Service Industry Mgmt 2003;14:232-44.

14 Egbu CO, Hari S, Renukappa SH. Knowledge management for sustainable competitiveness in small and medium surveying practices. Structural Survey 2005;23:7-21

15 Shelton RC, Cooper BR, Stirman SW. The sustainability of evidencebased interventions and practices in public health and health care. Annu Rev Public Health 2018;39:55-76.

16 Iwelunmor J, Blackstone S, Veira D. Toward the sustainability of health interventions implemented in sub-Saharan Africa : a systematic review and conceptual framework. Implement Sci 2016;11:1-27.

17 Mansour M, Mansour JB, Swesy AHE. Scaling up proven public health interventions through a locally owned and sustained leadership development programme in rural upper Egypt. Hum Resour Health 2010;8:1.

18 O'Neil M, Seims LRK, Cheburet S, et al. Leadership and management to empower the health workforce. In: Transforming the global health workforce. New York: New York University, College of Nursing, 2013: 225.

19 Seims LRK, Alegre JC, Murei L, et al. Strengthening management and leadership practices to increase health-service delivery in Kenya: an evidence-based approach. Hum Resour Health 2012;10:25.

20 Gruen RL, Elliott JH, Nolan ML, et al. Sustainability science: an integrated approach for health-programme planning. Lancet 2008;372:1579-89.

21 Sridharan S, Go S, Zinzow H, et al. Analysis of strategic plans to assess planning for sustainability of comprehensive community initiatives. Eval Program Plann 2007;30:105-13.

22 Pluye P, Potvin L, Denis J-L. Corrigendum to 'Making public health programs last: conceptualizing sustainability' [Evaluation and Program Planning 27 (2004) 121-133]. Eval Program Plann 2004;27:453.

23 Management Sciences for Health. Leadership can be learned, but how is it measured? occasional. Cambridge, MA. USA, 2008: 1-29. https://www.msh.org/sites/msh.org/files/how_can_leadership_be_ measured.pdf

24 World Health Organisation. Monitoring the building blocks of health systems: a Handbook of indicators and their measurements strategies. Geneva: World Health Organisation, 2010.

25 Baldwin TT, Ford JK. Transfer of training: a review and directions for future research. Pers Psychol 1988;41:63-105.

26 Seddiq K, Enarson DA, Shah K, et al. Implementing a successful tuberculosis programme within primary care services in a conflict area using the stop TB strategy: Afghanistan case study. Confl Health 2014;8:3-9.

27 Kwamie A, van Dijk H, Agyepong IA. Advancing the application of systems thinking in health: realist evaluation of the leadership development programme for district manager decision-making in Ghana. Health Res Policy Syst 2014;12:29.

28 Peterson EA, Dwyer J, Howze-Shiplett M, et al. Presence of leadership and management in global health programs: compendium of case studies. Washington DC: The George Washington University, 2011.

29 Chelagat T, Onyango J, Kokwaro G, et al. From strategy to action : a qualitative study on salient factors influencing knowledge transfer in project-based experiential learning in healthcare organisations in Kenya. BMJ Open 2019:1-12.

30 Stetler CB, Ritchie JA, Rycroft-Malone J, et al. Institutionalizing evidence-based practice: an organizational case study using a mode of strategic change. Implementation Sci 2009;4:1-19.

31 Collins D. Pretesting survey instruments: an overview of cognitive methods. Qual Life Res 2003;12:229-38.

32 Gillham B. Research interviewing: the range of techniques: a practical guide. Pennsylvania: McGraw-Hill Education (UK), 2005: 119-57.

33 Gale NK, Heath G, Cameron E, et al. Using the framework method for the analysis of qualitative data in multi-disciplinary health research. BMC Med Res Methodol 2013;13:1-8.

34 O'Brien BC, Harris IB, Beckman TJ, et al. Standards for reporting qualitative research: a synthesis of recommendations. Acad Med 2014;89:1245-51. 